\title{
Quantity not sufficient rates and delays in sweat testing in US infants with cystic fibrosis
}

\author{
Susanna McColley ${ }^{1}$, Alexander Elbert ${ }^{2}$, Runyu $\mathrm{Wu}^{2}$, Clement Ren ${ }^{3}$, Marci Sontag ${ }^{4}$, and V. \\ Legrys $^{5}$
}

${ }^{1}$ Ann \& Robert H.Lurie Children's Hospital of Chicago

${ }^{2}$ Cystic Fibrosis Foundation

${ }^{3}$ Indiana University System

${ }^{4} \mathrm{CI}$ International

${ }^{5} \mathrm{UNC}$

June 4, 2020

\begin{abstract}
Background: Diagnostic sweat testing is required for infants with positive newborn screening (NBS) tests for cystic fibrosis (CF). Infants have "quantity not sufficient" (QNS) sweat volumes more often than older children. A comprehensive study of QNS sweat volumes in infants has not previously been reported. Methods: We surveyed US CF Centers to obtain QNS rates in all infants who had sweat testing at $<14$ days and $<3$ months of age. We then calculated QNS rates reported to the Cystic Fibrosis Foundation Patient Registry (CFFPR) 2010-2018 in 10-day increments from 1 to 60 days of life. We compared QNS sweat tests rates in preterm $(<37$ weeks gestational age) versus term infants. We assessed age at sweat test and proportion of infants who did not have a sweat test reported by 60 days of age. Results: Thirty-nine of 144 (27\%) of CF Centers reported a mean QNS rate of $10.5 \%$ (range, 0-100) in infants < 14 days old. CFFPR data showed highest QNS rates in the youngest infants and in those born $<37$ weeks gestation. The median age at sweat testing decreased over time, but $>22 \%$ of infants did not have a sweat test reported by 60 days. Conclusion: Higher QNS rates are seen in the youngest infants with CF, but $>$ $80 \%$ of infants $<2$ weeks of age have adequate sweat volumes. Sweat testing should not be delayed in infants with a positive CF NBS test.
\end{abstract}

Quantity not sufficient rates and delays in sweat testing in US infants with cystic fibrosis

Susanna A. McColley, MD ${ }^{1}$; Alexander Elbert, $\mathrm{PhD}^{2}$; Runyu Wu, MS${ }^{2}$; Clement L. Ren, MD ${ }^{3}$ Marci K. Sontag, $\mathrm{PhD}^{4}$; Vicky A. LeGrys, DA, MT(ASCP) ${ }^{5}$.

1. Department of Pediatrics, Northwestern University Feinberg School of Medicine; Stanley Manne Children's Research Institute, Ann \& Robert H. Lurie Children's Hospital of Chicago, Chicago, IL.

2. Cystic Fibrosis Foundation, Bethesda, MD.

3. Department of Pediatrics, Indiana University; Riley Children's Hospital, Indianapolis, Indiana.

4. Center for Public Health Innovation, Littleton, Colorado; Department of Epidemiology, University of Colorado, Denver.

5. Department of Allied Health Science, University of North Carolina, Chapel Hill, NC.

Abstract

Background: Diagnostic sweat testing is required for infants with positive newborn screening (NBS) tests for cystic fibrosis (CF). Infants have "quantity not sufficient" (QNS) sweat volumes more often than older children. A comprehensive study of QNS sweat volumes in infants has not previously been reported. Methods: 
We surveyed US CF Centers to obtain QNS rates in all infants who had sweat testing at $<14$ days and $<3$ months of age. We then calculated QNS rates reported to the Cystic Fibrosis Foundation Patient Registry (CFFPR) 2010-2018 in 10-day increments from 1 to 60 days of life. We compared QNS sweat tests rates in preterm $(<37$ weeks gestational age) versus term infants. We assessed age at sweat test and proportion of infants who did not have a sweat test reported by 60 days of age. Results: Thirty-nine of $144(27 \%)$ of CF Centers reported a mean QNS rate of $10.5 \%$ (range, 0-100) in infants $<14$ days old. CFFPR data showed highest QNS rates in the youngest infants and in those born $<37$ weeks gestation. The median age at sweat testing decreased over time, but $>22 \%$ of infants did not have a sweat test reported by 60 days. Conclusion: Higher QNS rates are seen in the youngest infants with CF, but $>80 \%$ of infants $<2$ weeks of age have adequate sweat volumes. Sweat testing should not be delayed in infants with a positive CF NBS test.

Introduction

During the last decade, cystic fibrosis (CF) newborn screening (NBS) has been adopted throughout the United States (US) and in many other nations and regions. A positive NBS result may be reported before 2 weeks of age when a single dried blood spot is used for screening. Quantitative pilocarpine iontophoresis sweat chloride testing is recommended for evaluation of infants with a positive $\mathrm{NBS}^{1}$ and guidelines have been updated to enhance sweat testing in infants ${ }^{2}$. However, young infants are at increased risk of having quantity not sufficient (QNS) sweat volumes (or weights) compared to older infants and children ${ }^{3-5}$.

Surveys of QNS rates in US CF Care Centers led to guidance that CF Centers should have quantity not sufficient rates of $<10 \%$ in infants under 3 months of age and $<5 \%$ in older patients ${ }^{6}$. These data were collected before CF NBS was implemented in all states. Risk factors for QNS sweat tests in infants include gestational age $<38$ weeks, postnatal age $<14$ days, and weight $<2 \mathrm{~kg}\left({ }^{3-5}\right.$. Variability in QNS rates and high QNS rates in infants have been observed in the US ${ }^{6}$ and Europe ${ }^{7}$. We hypothesized that sweat testing QNS rates are higher in infants less than 14 days old or less than 37 weeks gestational age. We evaluated QNS rates and age at sweat testing in infants in the US seen at Cystic Fibrosis Foundation (CFF) -accredited Care Centers after initiation of NBS in all US states, utilizing a survey of CF Center Directors and data from the Cystic Fibrosis Foundation Patient Registry (CFFPR). We also evaluated delays in sweat testing in infants reported to the CFFPR.

Methods

For preliminary data, we surveyed Cystic Fibrosis Foundation accredited CF Center pediatric and affiliate program directors to estimate QNS rates in infants presenting for evaluation of a positive NBS test. Based on prior literature, we aimed to assess rates for infants who were $<30$ days old, and of the subset who were $<14$ days old, at time of sweat testing. The 6 -question survey (table 1) was sent via email directly from the CFF to 144 pediatric and affiliate program directors by the CFF. Participation was encouraged, but voluntary. This study was deemed exempt by the Ann \& Robert H. Lurie Children's Hospital of Chicago Institutional Review Board.

Based on our initial findings, we evaluated data submitted to CFFPR from January 1 2010-December 31 2018 to calculate sweat test QNS rates in infants with CF who had a sweat test recorded before 60 days of age. The CFFPR structure has been previously described (Knapp). The year 2010 was chosen since it was the first full year in which all US states screened infants for CF. Rates of QNS sweat tests were calculated for infants in 10-day increments from 0- $<10$ days to 50-60 days. We also compared QNS rates for infants born at 37 weeks (pre-term) to those born $>37$ weeks gestation (term). Finally, we compared data submitted to the CFFPR to evaluate the age at first sweat test for infants in three time periods: 2010-2012, 2013-2015, and 2016-2018. Data entry into the CFF patient registry is approved by local or central institutional review boards, and legal guardians of minors provide informed consent for participation.

Statistical analysis

Descriptive statistics were generated for the survey questions. Changes in the median age at sweat test were compared using the Wilcoxon rank sum test. Comparisons of QNS rates across postnatal age and gestational 
age categories was compared using the Chi-square test. $\mathrm{P}<.05$ was considered statistically significant.

Results

The CF Center survey, conducted between June 6 and July 4 2016, was completed by 39 (27\%) of CF center directors (table 2). There was great variability in the number of sweat tests performed by responding programs. Infants $<14$ days of age comprised a minority of those tested, and one third of programs had no tests performed in infants in this age group. In infants $<14$ days, the mean QNS rate was 10.5 (SD $=$ $22.2 \%$; range, $0-100 \%)$.

During the time period from January 1, 2010 and December 31, 2018, 5392 infants with CF were reported in the CFFPR. The QNS rate in infants $<10$ days of age was $16.4 \%$. With each 10-day increment, the QNS rate decreased (table 3). There were 3418 infants born $>37$ weeks gestation and 287 infants born $<37$ weeks gestation. Quantity not sufficient sweat tests were reported in $224(6.6 \%)$ term infants and 56 (19.5\%) of preterm infants $(\mathrm{p}<0.001$, Chi-square test). Variation by gestational age or age at sweat test were not evaluated due to the small number of preterm infants. There was a decrease in median age at sweat test over time (table 5). Among infants reported, 1204 (23.8\%) had no sweat test reported before 60 days of age. The percentage of patients with no sweat test before 60 days decreased slightly over time.

\section{Discussion}

This is the first large study of sweat test QNS rates and delayed sweat testing in infants and are important for center assessment of sweat testing, quality improvement efforts, and communicating with parents of infants with positive newborn screening tests. Newborn screening identifies infants at risk for CF. Prompt diagnostic testing is critical to identify infants with $\mathrm{CF}$ and to resolve the diagnosis in infants who do not have $\mathrm{CF}$. Delays in diagnostic confirmation are associated with parental psychological distress ${ }^{8}$ and in treatment. The quantitative pilocarpine iontophoresis sweat test is recommended for CF diagnosis ${ }^{9,10}$. It confirms CFTR dysfunction, has a rapid turnaround time, and is widely available.

The center director survey provides evidence that QNS rates are higher in younger infants and confirms previous findings that there is high variability in QNS rates between CF Centers ${ }^{6}$. Because only $27 \%$ of CF centers responded, the overall population QNS rates cannot be adequately estimated from these data. Data may not be representative of CF Centers, since those with better rates may have been more likely to respond. However, the large number and broad range of QNS results show significant variability in sweat test performance and include QNS rates in infants with and without CF.

Consistent with other studies ${ }^{3-5}$, we found that QNS rates are highest in infants during the first 10 days and in infants born prematurely. A novel finding is that sweat test QNS rate decreased over each subsequent 10-day increment. We did not evaluate infant weight at the time of sweat testing as it is not available in the registry. We did not assess other potential factors influencing QNS rates that have been inconsistent between studies. These include sweat collection methodology ${ }^{4,11}$ and African-American race ${ }^{3,4}$. Some infants in the CFFPR may have had QNS sweat tests that were not entered into the registry, underestimating the overall QNS rates. Therefore, we caution against using these rates to set QNS goals for quality assurance until additional prospective data are collected.

We found that while the median and mean age of sweat testing decreased over time, more than 1:5 infants seen at US CFF Care Centers during 2010-2018 did not have a sweat test by 60 days of age. This may be due to intentional delays in infants who have two CFTR mutations reported on NBS or by prenatal testing. However, some infants with two mutations may not have $\mathrm{CF}^{12}$, and there is a theoretical risk of NBS specimens being misattributed during collection or in a laboratory. Since most infants $<10$ days old or $<37$ weeks gestation had sufficient sweat quantities, sweat testing should not be delayed because of chronological or gestational age if the infant is physiologically stable and large enough for the test. Those who have a QNS test or are unable to undergo sweat testing due to illness or small size should be evaluated for signs and symptoms of CF. Empiric therapy with sodium chloride and pancreatic enzymes should be considered in these circumstances and later withdrawn if the sweat test is normal. Additional testing, such 
as fecal pancreatic elastase or confirmatory or expanded CFTR genotyping on a sample from the infant, may also be useful if sweat tests are persistently QNS.

Due to the potential that CF Centers or their associated laboratories might discourage testing in young infants fearing an increased QNS rate, the CFF has modified the reporting system for accredited centers. Effective 2019, QNS rates are being evaluated for patients between 6 weeks to 3 months of age with a benchmark of $10 \%$ and patients over 3 months of age with a benchmark of $5 \%$. QNS rates in patients less than 6 weeks of age will be collected and evaluated for establishment of an appropriate standard.

Finally, even with sweat test improvement efforts, a proportion of infants with positive NBS tests will have a QNS sweat test. Guidelines for diagnosis of CF in screened populations ${ }^{9}$ recommend prompt evaluation of infants with a positive NBS test and presumptive treatment of infants who have signs and symptoms, including poor growth or abnormal stools, to reduce the risk of growth failure, hyponatremia and other complications. We further recommend presumptive treatment in infants with 2 copies of disease causing CFTR mutations. For infants at risk for pancreatic insufficiency, treatment with pancreatic enzyme replacement therapy has few side effects and may be lifesaving. Sodium chloride supplementation is low cost, widely available in the form of table salt, and can avoid life-threatening hyponatremia.

Conclusion: Young and premature infants have higher QNS sweat test rates than older infants. Sweat testing should not be delayed and is often successful even before 10 days of age. Evaluation of sweat collection proficiency should account for the proportion of young and premature infants evaluated and requires expansion beyond this report. Regardless of the ultimate standard set for QNS rates, ongoing quality improvement work should be undertaken to minimize sweat test QNS rates. This will improve timing of diagnostic resolution and benefit infants with positive NBS tests, their families, and the healthcare system.

Funding sources: This work was funded by Cystic Fibrosis Foundation grants MCCOLL15QI0 and MCCOLL19QI0.

References:

1. LeGrys VA, Yankaskas JR, Quittell LM, Marshall BC, Mogayzel PJ, Foundation CF. Diagnostic sweat testing: the Cystic Fibrosis Foundation guidelines. J Pediatr 2007;151(1):85-9.

2. CLSI. Sweat Testing: Specimen Collection and Quantitative Chloride Analysis. 4th ed. CLSI guideline C34. Wayne, PA: Clinical and Laboratory Standards Institute; 2019.

3. Kleyn M, Korzeniewski S, Grigorescu V, Young W, Homnick D, Goldstein-Filbrun A, Schuen J, Nasr S. Predictors of insufficient sweat production during confirmatory testing for cystic fibrosis. Pediatr Pulmonol 2011;46(1):23-30.

4. Eng W, LeGrys VA, Schechter MS, Laughon MM, Barker PM. Sweat-testing in preterm and full-term infants less than 6 weeks of age. Pediatr Pulmonol 2005;40(1):64-7.

5. Collins MN, Brawley CB, McCracken CE, Shankar PR, Schechter MS, Rogers BB. Risk factors for quantity not sufficient sweat collection in infants 3 months or younger. Am J Clin Pathol 2014;142(1):72-5.

6. Legrys VA, McColley SA, Li Z, Farrell PM. The need for quality improvement in sweat testing infants after newborn screening for cystic fibrosis. J Pediatr 2010;157(6):1035-7.

7. Cirilli N, Southern KW, Buzzetti R, Barben J, Nährlich L, Munck A, Wilschanski M, De Boeck K, Derichs N, Group otEDNW. Real life practice of sweat testing in Europe. J Cyst Fibros 2017.

8. Tluczek A, Koscik RL, Farrell PM, Rock MJ. Psychosocial risk associated with newborn screening for cystic fibrosis: parents' experience while awaiting the sweat-test appointment. Pediatrics 2005;115(6):1692703. 
9. Farrell PM, White TB, Howenstine MS, Munck A, Parad RB, Rosenfeld M, Sommerburg O, Accurso FJ, Davies JC, Rock MJ and others. Diagnosis of Cystic Fibrosis in Screened Populations. J Pediatr 2017;181S:S33-S44.e2.

10. Farrell PM, White TB, Ren CL, Hempstead SE, Accurso F, Derichs N, Howenstine M, McColley SA, Rock M, Rosenfeld M and others. Diagnosis of Cystic Fibrosis: Consensus Guidelines from the Cystic Fibrosis Foundation. J Pediatr 2017;181S:S4-S15.e1.

11. Laguna TA, Lin N, Wang Q, Holme B, McNamara J, Regelmann WE. Comparison of quantitative sweat chloride methods after positive newborn screen for cystic fibrosis. Pediatr Pulmonol 2012;47(8):736-42.

12. Bergougnoux A, Boureau-Wirth A, Rouzier C, Altieri JP, Verneau F, Larrieu L, Koenig M, Claustres M, Raynal C. A false positive newborn screening result due to a complex allele carrying two frequent CF-causing variants. J Cyst Fibros 2016;15(3):309-12.

\section{Hosted file}

Table 1.docx available at https://authorea.com/users/329800/articles/456715-quantity-notsufficient-rates-and-delays-in-sweat-testing-in-us-infants-with-cystic-fibrosis

\section{Hosted file}

Table 2.docx available at https://authorea.com/users/329800/articles/456715-quantity-notsufficient-rates-and-delays-in-sweat-testing-in-us-infants-with-cystic-fibrosis

\section{Hosted file}

Table 3.docx available at https://authorea.com/users/329800/articles/456715-quantity-notsufficient-rates-and-delays-in-sweat-testing-in-us-infants-with-cystic-fibrosis

\section{Hosted file}

Table 4.docx available at https://authorea.com/users/329800/articles/456715-quantity-notsufficient-rates-and-delays-in-sweat-testing-in-us-infants-with-cystic-fibrosis 